\title{
La photographie documentaire contemporaine dans les Amériques
}

Contemporary documentary photography in the Americas

Fotografía documental contemporánea en las Américas

\section{Marion Gautreau et Jean Kempf}

\section{OpenEdition}

\section{Journals}

Édition électronique

URL : http://journals.openedition.org/ideas/5099

DOI : 10.4000/ideas.5099

ISSN : 1950-5701

\section{Éditeur}

Institut des Amériques

\section{Référence électronique}

Marion Gautreau et Jean Kempf, « La photographie documentaire contemporaine dans les Amériques », IdeAs [En ligne], 13 | 2019, mis en ligne le 01 mars 2019, consulté le 24 septembre 2020. URL http://journals.openedition.org/ideas/5099 ; DOI : https://doi.org/10.4000/ideas.5099

Ce document a été généré automatiquement le 24 septembre 2020.

\section{(i) $\ominus$

IdeAs - Idées d'Amériques est mis à disposition selon les termes de la licence Creative Commons Attribution - Pas d'Utilisation Commerciale - Pas de Modification 4.0 International. 


\title{
La photographie documentaire contemporaine dans les Amériques
}

\author{
Contemporary documentary photography in the Americas \\ Fotografía documental contemporánea en las Américas
}

Marion Gautreau et Jean Kempf

1 Il est banal de dire, mais faut-il encore le rappeler, que les liens entre la photographie et le monde réel sont d'une nature particulière en raison de la manière dont l'image est produite. Et c'est cette nature particulière qui très tôt en a fait un outil de dévoilement et de connaissance du monde. Ce qui s'est petit à petit défini comme «le documentaire » (on s'accorde en général pour placer l'apparition du terme dans les années 1920-1930) s'ancre dans cette modalité de l'image photographique. Il s'agit d'utiliser la photographie non comme un mode d'expression mais au contraire d'exploration. Pourtant, quoique simple en apparence, cette opposition est bien plus didactique que réelle - aucune frontière nette entre les deux domaines. Mais s'il existe de nombreuses manières d'utiliser la photographie dans son lien au «réel »-au hasard, la photographie familiale, la photographie scientifique, de presse, d'identité ou policière - le documentaire est d'une autre nature. Certes proche du photojournalisme voire de la photographie de presse, à la fois par les thèmes abordés et parfois par la forme plastique, il ne saurait pour autant se confondre avec lui. Le documentaire ne se définit ni par son style comme le proposait Olivier Lugon dans un propos historique (Lugon O., 2001), ni par son sujet, l'extraordinaire, comme l'écrivait Stuart Franklin (Franklin S., 2016: 9). Ses caractéristiques sont, d'une part, un propos, une intention, une position d'auteur et, d'autre part, une relation entre photographe et sujet qui implique la durée (même si les temporalités peuvent être très variables, de quelques jours à plusieurs années voire décennies). Ces deux modalités impliquent donc aussi une attention particulière portée à la manière dont le documentaire va être diffusé : formes appropriées à une narration complexe et très grande vigilance du (de la) photographe à l'usage qui en est ou sera fait. Cet ensemble de contraintes peut se résumer par le mot « exigence». 
2 Rebeca Monroy Nasr, historienne de la photographie mexicaine depuis plus de trente ans et photographe à ses débuts, propose, dans l'introduction de son dernier ouvrage, de différencier ainsi photojournalisme et photographie documentaire, deux pratiques qui ont en commun une visée informative mais dont le mode de réalisation et les usages diffèrent grandement :

La différence entre la photographie documentaire et le photojournalisme s'établit à partir de l'usage social de l'image et de l'intention avec laquelle le photographe prend cette image. Nous nous considérions des photographes documentaires car nous capturions un événement sans avoir la certitude de la vente ou de la publication de ce matériau. Nous travaillions avec le désir de léguer un témoignage visuel du moment, même si c'était pour qu'il dorme dans nos archives personnelles; nous espérions la possibilité de publier ou bien d'exposer ou éditer ces photographies dans un espace public, à plus long terme (Monroy Nasr R., 2017 : 21) 1 .

Des grandes opérations photographiques qui documentèrent les expéditions dans l'Ouest étasunien (surveys) au XIX siècle aux enquêtes urbaines sur le mal logement, l'immigration et le travail des enfants au tournant du siècle (Jacob Riis et Lewis Hine par exemple), aux images, enfin, de la Farm Security Administration dans les années 1930, la démarche documentaire jouit déjà d'un long passé lorsqu'elle est reprise et utilisée, à partir des années 1920 puis surtout des années 1930 à 1960, par la presse illustrée sous la forme de l'essai photographique - photo essay en anglais, fotoensayo en espagnol (Chouard G. et al., 2014). Ce terme, que nous pourrions traduire par « essai photographique » mais qui est très peu utilisé en français, reprend les codes de l'essai littéraire : proposer une réflexion structurée autour d'un sujet clairement délimité afin de susciter une prise de conscience et d'engager, éventuellement, un débat autour de ce sujet. C'est dans ce cadre-là que certains photojournalistes adoptent dans leur profession la pratique du "double appareil photo " («doble cámara », en espagnol). Le premier appareil sert à faire des photographies destinées aux journaux et aux sites Internet d'information; il produit des images permettant de voir le mieux possible le contenu informatif de la situation. Le deuxième appareil est utilisé pour produire des images qui vont s'insérer dans des séries photographiques, pensées comme telles et menées sur le long terme. C'est le cas par exemple de Fernando Brito, photojournaliste mexicain de l'État du Sinaloa, qui dénonce à travers son travail documentaire les violences liées à la guerre du narcotrafic, développant ainsi une conscience sociale intrinsèquement liée au documentaire.

4 On le voit, si la domination des modèles étasuniens sur le monde de la photographie depuis au moins la fin de la Seconde Guerre mondiale est avérée, et non pas seulement dans le domaine de la photographie d'art (ou plasticienne), il subsiste, même en cette époque de mondialisation des images, des cultures visuelles spécifiques - locales - qui appellent ou simplement produisent des formes particulières de narration visuelle. Toute la difficulté, et tout l'intérêt, de penser le documentaire photographique depuis le continent américain dans son entièreté, provient des différences de temporalités dans le développement de cette pratique photographique selon les régions. Par ailleurs, l'histoire de la photographie ayant été principalement écrite et diffusée depuis l'Europe et les États-Unis, l'exhumation de l'histoire de la photographie documentaire dans les pays d'Amérique latine relève encore parfois de la recherche archéologique. Peu de récits d'historiens décrivent la généalogie du documentaire chilien ou brésilien même si, d'évidence, cette lignée existe malgré son absence de tracé, alors que du côté nord- 
américain, depuis au moins l'article de Beaumont Newhall de 1938 puis la première édition de son histoire de la photographie (1949), le genre est repéré, décrit, codifié et en partie aussi encadré et normé 2 .

5 De plus, il n'existe pas, a priori, de convergence entre les différentes phases de la photographie documentaire au nord et au sud du continent, comme il n'existe pas non plus de convergence entre les "différents suds» du continent. Si les techniques se diffusent de la même manière dans l'ensemble des Amériques (apparition de la similigravure, généralisation de la pellicule souple, introduction de la couleur, passage à la photographie numérique), en revanche, l'impulsion donnée à la photographie documentaire par les institutions et contextes politiques et sociaux ainsi que par les possibilités de productions, diffère sensiblement selon les pays. Les dictatures du Cône sud dans les années 1970 et 1980 révèlent à la fois un besoin criant d'essai photographique sur cette période de répression et une impossibilité matérielle et physique de le mener à bien ${ }^{3}$. À la même période, au contraire, se développe au Mexique une intense activité documentaire qui prend naissance dans le photojournalisme, en particulier à travers les journaux Unomásuno et La Jornada, mais dont les photographes vont peu à peu s'émanciper pour lui préférer le temps long.

C'est pour cette raison que nous avons choisi de demander à des spécialistes de la photographie et des cultures des Amériques de réfléchir sur des pratiques documentaires spécifiques afin d'en montrer la diversité, la richesse et la complexité, plus que la convergence.

7 Ce numéro sur des pratiques documentaires dans les Amériques (aucune exhaustivité n'est bien entendu possible) s'organise cependant autour de trois modalités que l'on retrouve à des degrés divers dans nombre de projets documentaires: la référence scientifique ou au contraire idéologique; la forme artistique comme accès aux complexités du réel ; et enfin l'usage du documentaire comme outil d'affirmation d'une identité ou d'une reconquête de mémoire.

8 Combinant l'observation de longue durée et la narration, le documentaire fait naturellement et largement usage des séries selon des formes plus ou moins strictes mais qui toujours dilatent la dimension temporelle pour en saisir le sens dans le processus de transformation. C'est le cas du méticuleux travail d'inventaire de Camilo José Vergara, un photographe chilien qui a travaillé aux États-Unis où il a compilé une archive urbanistique méthodique de ghettos noirs dans lequel Philippe Bazin (lui-même photographe) voit une machine de guerre au sens deleuzien, "un moyen de faire sortir [le ghetto] du carcan dans lequel l'ultralibéralisme violent l'a enfermé depuis des décennies ».

9 Tout aussi engagées, mais presque aux antipodes de la forme, les images que réalise le Suisse Jean-Claude Wicky entre 1984 et 2001 dans des mines boliviennes sont un exemple à la fois d'une photographie humaniste classique qui rappelle, avec ses corps rendus dans de riches tirages noir-et-blanc, les images de W. Eugene Smith, et de l'observation participative. Baptiste Lavat nous présente, avec le travail peu connu en France de Jean-Claude Wicky, ce qui constitue l'expérience de nombre de documentaristes au quotidien : le partage de vies qui ne sont plus tout à fait celles de leurs sujets sans pourtant jamais complètement être la leur, un entre-deux difficile que connaissent aussi les ethnologues, voire les sociologues.

C'est donc un contraste brutal qui attend le lecteur qui suivrait l'ordre du sommaire pour découvrir ensuite le spectacle de la catastrophe, comme Danièle Méaux nomme 
les images des «ruines de Detroit » de Yves Marchand et Romain Meffre, et le travail sophistiqué et ambigu de Joel Sternfeld sur la High Line de New York. Les vestiges d'un passé industriel puissant suscitent aujourd'hui un engouement pour ce que l'auteure appelle d'une jolie formule «le néo-pittoresque déglingué» qu'exploite une photographie post-moderne; malgré des images qui stimulent l'imagination des lieux et proposent « un paysage en devenir », Joel Sternfeld, avec la documentation même de la transformation paysagère du passé industriel, participe de la réappropriation chic par des classes sociales privilégiées des anciens espaces de travail et de production devenus de loisir.

11 Cette difficulté à dire la brutalité et l'exclusion que génère la ville moderne, et plus généralement à dire le réel tout court (dans son aspect le plus cru), est analysé par Gwen Cressman qui a choisi pour cela de comparer deux œuvres photographiques très différentes, celles de Martha Rosler et de Jeff Wall, mais qui partagent une même méfiance vis-à-vis de la possibilité d'un accès simple et direct au réel par la photographie. Plasticiennes et conceptuelles, leurs photographies ne cessent d'interroger ce désir de voir et cette résistance du monde à se laisser comprendre.

Beaucoup plus concrète, la troisième partie de cette livraison propose trois parcours différents qui combinent à la fois le témoignage sur une ou des réalités sociales, culturelles ou historiques, et une tentative de déconstruction des stéréotypes à travers non seulement la fabrication des images mais aussi l'intégration à des ensembles discursifs, à des dispositifs spécifiques. Morgana Herrera présente le travail de Musuk Nolte, un photographe péruvien qui, à travers ses séries en noir-et-blanc, se place à contre-courant des représentations exotiques de l'espace amazonien. Grâce au choix de ses sujets (plus urbains que ruraux) et au traitement des personnes photographiées (dans leur quotidien et les contraintes de leurs conditions de vie), il offre une place non-ethnographique aux habitants de l'Amazonie péruvienne sur la scène visuelle internationale. Maude Oswald, quant à elle, montre comment un inventaire de signes fonctionnels (les marques de la défense civile sur les bâtiments abandonnés après l'ouragan Katrina) devient sur le web un outil de construction mémoriel a posteriori, signifiant ainsi l'action « en retour » des photographes sur le réel sous forme d'une ré-appropriation. Cette ré-appropriation est au centre du projet que se propose d'étudier Aurélie Journée, celui d'une photographe amérindienne dont les portraits essaient de combiner l'héritage ancestral et une modernité qui peut faire advenir une parole plus épanouie, en particulier des femmes autochtones.

On le voit, si le parcours proposé est nécessairement partiel, il rend cependant compte des subtiles différences au sein d'une préoccupation qui traverse le documentaire : celle de l'émancipation. 


\section{BIBLIOGRAPHIE}

Chouard, Géraldine, Kempf, Jean et Brunet, François, « La photographie "documentaire” américaine : nouvelles approches », Transatlantica, vol. 2, 2014, http://journals.openedition.org/ transatlantica/7245, page consultée le 23 février 2019.

Franklin, Stuart, The Documentary Impulse, Londres, Phaidon, 2016.

Lugon, Olivier, Le style documentaire : d'August Sander à Walker Evans, 1920-1945, Paris, Macula, 2001.

Meiselas, Susan (dir.), Chile from within, New York ; Londres, W.W. Norton \& Company, 1990.

Monroy Nasr, Rebeca, Con el deseo en la piel. Un episodio de la fotografía documental a fines del siglo XX, Mexico, UAM-Xochimilco, 2017.

Newhall, Beaumont, « Documentary Approach to Photography », Parnassus, vol. 10, n 3, 1938, p. 2-6 [accessible sur JSTOR, http://www.jstor.org/stable/771747].

Newhall, Beaumont, The History of Photography from 1839 to the Present Day, New York, Museum of Modern Art, 1949. Traduction française en 1967.

\section{NOTES}

1. «La diferencia entre fotodocumentalismo y fotoperiodismo se establece a partir del uso social de la imagen y la intención con la que el fotógrafo toma esta imagen. Nos considerábamos fotógrafos documentales aquellos que captábamos el acontecimiento sin tener la certeza clara de la venta o la publicación del material. Trabajábamos con el deseo de dejar un testimonio visual del momento aunque éste solo se integrara a nuestro acervo personal, con la posibilidad de publicar posteriormente para realizar alguna exposición o edición de las imágenes en algún espacio público. "

2. On peut cependant citer les noms d'historiens de la photographie latino-américains qui, à l'heure actuelle, reconstruisent ces récits petit à petit: Cora Gamarnik en Argentine, Boris Kossoy et Ana María Mauad au Brésil, Rebeca Monroy Nasr, John Mraz ou Alberto del Castillo au Mexique, pour n'en citer que quelques-uns.

3. Voir à ce propos Meiselas S., 1990.

\section{AUTEURS}

\section{MARION GAUTREAU}

Enseignante-chercheuse au département d'études hispaniques et hispano-américaines de l'Université Toulouse-Jean Jaurès. Historienne de la photographie latino-américaine, elle est notamment spécialiste du Mexique. Ses recherches portent essentiellement sur la photographie de presse et la photographie documentaire dans leur articulation avec le récit historique 
national, aux $\mathrm{xx}^{\mathrm{e}}$ et $\mathrm{xxI}^{\mathrm{e}}$ siècles. Elle a publié un ouvrage sur la photographie de la Révolution mexicaine, De la crónica al ícono: la fotografía de la Revolución Mexicana en la prensa ilustrada capitalina (1910-1940), et travaille actuellement sur le photojournalisme mexicain depuis 1968.

\section{JEAN KEMPF}

Professeur de civilisation des États-Unis à l'Université Lumière-Lyon 2. Il est spécialiste de l'histoire de la photographie américaine au $\mathrm{xx}^{\mathrm{e}}$ siècle. Il a notamment écrit sur la commande institutionnelle, sur les street photographers et sur les pratiques documentaires, et s'intéresse aujourd'hui à la sociologie des milieux photographiques contemporains. Il a participé à l'aventure de L'Amérique des images (Hazan, 2013) et a publié une Histoire culturelle des États-Unis (Belin, 2015). 